\title{
Avaliação de método para reconstrução acetabular com uso de enxerto ósseo homólogo e implante cimentado*
}

\author{
Evaluation of a method of acetabular reconstruction \\ using homologous bone graft and cemented implant
}

\author{
Milton Valdomiro RoOs ${ }^{1}$, BRUno Dutra RoOs², \\ Carolina Monteiro Sampaio², PaUlo Roberto Maroues Junior ${ }^{2}$
}

\section{RESUMO}

Objetivo: Avaliar clínica e radiograficamente 43 quadris em 43 pacientes submetidos a tratamento cirúrgico, para reconstrução acetabular do quadril pós-afrouxamento asséptico do implante, utilizando classificação idealizada e adotada no Serviço de Cirurgia do Quadril do Pronto Socorro de Fraturas de Passo Fundo/RS (método dos $75 \mathrm{~mm}$ ). Métodos: Foi realizado estudo retrospectivo com 88 pacientes ( 90 quadris) submetidos a tratamento cirúrgico de reconstrução acetabular após afrouxamento asséptico do implante, entre agosto de 1994 e outubro de 2000. Desses pacientes, 43 (43 quadris) preencheram todos os requisitos necessários para este trabalho. Foi considerada falha da reconstrução devido a afrouxamento, a migração do implante maior do que $5 \mathrm{~mm}$ em qualquer direção, ou a progressão de linhas de radioluscência maior do que $2 \mathrm{~mm}$ de

* Trabalho realizado no Centro de Estudos Ortopédicos de Passo Fundo, Faculdade de Medicina da Universidade de Passo Fundo - UPF - Passo Fundo (RS), Brasil.

1. Coordenador do Programa de Residência Médica em Ortopedia e Traumatologia do Centro de Estudos Ortopédicos de Passo Fundo - CEOP - Passo Fundo (RS), Brasil.

2. Residentes do 3o Ano do Programa de Residência Médica do Centro de Estudos Ortopédicos de Passo Fundo - CEOP - Passo Fundo (RS), Brasil.

Endereço para correspondência: Bruno Dutra Roos, Av. Sete de Setembro, 817, Centro - 99010-121 - Passo Fundo (RS), Brasil. Tel./fax: (54) 2104-4333. E-mail: broos@ pop.com.br

Recebido em 3/7/08. Aprovado para publicação em 22/9/08. Copyright RBO2008 largura nas zonas delimitadas por DeLee e Charnley, em associação com o critério clínico de dor. $R \boldsymbol{e}$ sultados: Do total de pacientes avaliados, 29 casos $(67,4 \%)$ foram submetidos à reconstrução acetabular com enxerto picado tipo "crouton" $\left(1 \mathrm{~cm}^{3}\right)$; os 14 casos $(32,6 \%)$ restantes foram submetidos à reconstrução acetabular com enxerto em bloco. A classificação clínica pós-operatória, de acordo com os critérios de D'Aubignè et al modificados por Charnley, considerou os resultados pós-operatórios obtidos como: $83,7 \%$ satisfatórios e $16,3 \%$ insatisfatórios, com seguimento mínimo de seis anos. De acordo com os critérios estabelecidos, sete pacientes $(\mathbf{1 6 , 3 \%})$ apresentaram falhas de reconstrução devido a afrouxamento: seis com enxerto picado (20,7\% das 29 enxertias com osso picado) e uma com enxerto em bloco ( $7,1 \%$ dos 14 blocos). As falhas ocorreram, em média, aos 7,25 anos. Conclusão: Com base nos resultados obtidos, utilizando, como critério de indicação da técnica cirúrgica a adotar, a mensuração da cavidade acetabular préoperatória (maior ou menor do que $75 \mathrm{~mm}$ ), conclui-se que o método apresentou alto índice de sobrevida da reconstrução acetabular e resultados clínicos satisfatórios em seguimento médio de 8,3 anos. Constatou-se maior sobrevida nos casos que foram realizados utilizando blocos ósseos, apesar de a cavidade ser de maior dimensão.

\footnotetext{
Descritores - Artroplastia do quadril/métodos; Acetábulo/ cirurgia; Transplante homólogo/efeitos adversos; Falha de prótese
} 


\section{ABSTRACT}

Objective: To make a clinical and radiographic evaluation of 43 hips in 43 patients submitted to surgical treatment for acetabular reconstruction of the hip after aseptic loosening of the implant, using the classification developed and adopted by the Hip Surgery Service of the Fracture Emergency Unit in Passo Fundo, State of Rio Grande do Sul (Method of the $75 \mathrm{~mm}$ ). Methods: A retrospective study was performed with 88 patients (90 hips) submitted to surgical treatment for acetabular reconstruction after aseptic loosening of the implant, from August 1994 to October 2000. Out of those patients, 43 (43 hips) met all inclusion requirements for this study. Migration of an implant longer than $5 \mathrm{~mm}$ in any direction, or the progression of radiolucent lines of more than $2 \mathrm{~mm}$ in width in the zones limited by DeLee and Charnley, combined with pain were considered a failed reconstruction due to loosening. Results: From the total number of patients evaluated, 29 cases (67.4\%) were submitted to acetabular reconstruction with "crouton" plicated graft (1 $\left.\mathrm{cm}^{3}\right)$; the other 14 cases (32.6\%) were submitted to acetabular reconstruction with block graft. The post-operative clinical classification according to the criteria of D'Aubignè et al modified by Charnley, considered the results achieved as follows: $83.7 \%$ satisfactory, and $16.3 \%$ unsatisfactory, with a minimum follow-up of six years. According to the criteria established, seven patients $(16.3 \%)$ presented reconstruction failure due to loosening: six with the plicated graft $(20.7 \%$ of the 29 grafts with plicated bone) and one with block graft (7.1\% of the 14 block grafts). On the average, the failures occurred at 7.25 years. Conclusion: Based on the results achieved and using the measurement of the pre-operative acetabular cavity (bigger or smaller than $75 \mathrm{~mm}$ ) as the criterion of indication of the surgical technique to be adopted, the authors conclude that the method had a high rate of survival of the acetabular reconstruction and satisfactory clinical results in 8.3 years of mean follow up. A longer survival was observed in the cases in which bone blocks were used despite the cavity being bigger.

Keywords - Arthroplasty, replacement, hip/methods; Acetabulum/surgery; Transplantation, homologous/adverse effects; Prosthesis failure

\section{INTRODUÇÃO}

A complicação mais freqüente nas artroplastias de quadril, em longo prazo, é o afrouxamento asséptico do implante, com conseqüente reabsorção óssea ${ }^{(1)}$. A técnica cirúrgica ideal para a reconstrução acetabular permanece um desafio para os cirurgiões de quadril.

$\mathrm{Na}$ literatura são relatadas diferentes opções de tratamento, como o uso de enxerto picado isolado ou associado a reforços, enxerto estrutural, cimento ósseo, próteses de grandes dimensões e outros métodos para o preenchimento do defeito acetabular ${ }^{(2)}$. Contudo, apesar das várias estratégias cirúrgicas publicadas, ainda não há consenso sobre o melhor método a ser utilizado.

O objetivo do presente estudo é avaliar os resultados clínicos, radiográficos e a sobrevida do tratamento cirúrgico de reconstrução acetabular por afrouxamento asséptico do implante, utilizando classificação idealizada e adotada pelo Serviço de Cirurgia do Quadril do Pronto Socorro de Fraturas de Passo Fundo/RS (SCQ/ $\mathrm{PSF} / \mathrm{PF} / \mathrm{RS}$ ) (método dos $75 \mathrm{~mm}$ ).

\section{MÉTODOS}

Foi realizado estudo retrospectivo com 88 pacientes (90 quadris) submetidos a tratamento cirúrgico de reconstrução acetabular secundário a afrouxamento asséptico de artroplastia, de agosto de 1994 a outubro de 2000. Todas as reconstruções foram realizadas pela mesma equipe médica e com o método idealizado no próprio Serviço.

Foram incluídos no estudo pacientes com uma ou mais artroplastias prévias que apresentavam afrouxamento asséptico do implante, os quais foram submetidos à reconstrução utilizando acetábulo cimentado tipo Charnley associado a enxertia óssea homóloga.

Os critérios de exclusão foram: seguimento menor do que seis anos (31 casos), perdas de seguimento (sete casos), infecção pós-operatória (quatro casos), faleci- 

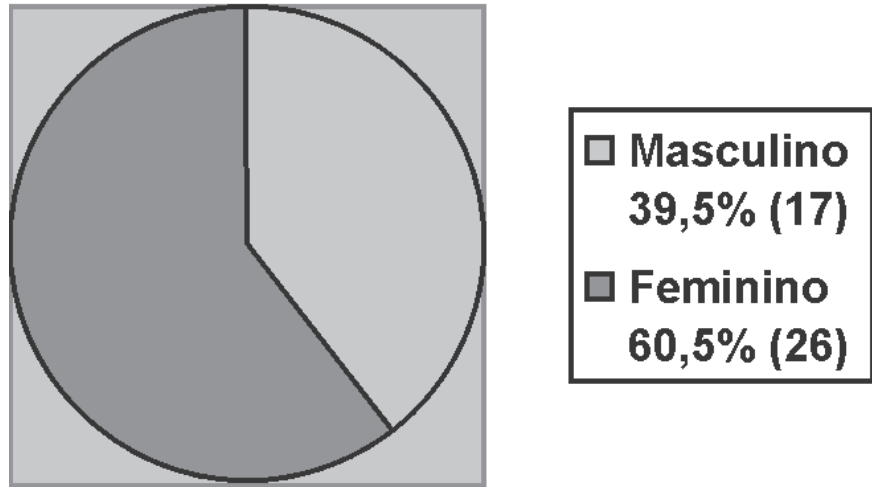

Gráfico 1 - Caracterização dos pacientes quanto ao sexo

mento antes do seguimento mínimo (três casos) e luxação recidivante de prótese (dois casos).

De acordo com os critérios estabelecidos, 43 pacientes (43 quadris) preencheram todos os requisitos necessários para este trabalho, sendo 26 do sexo feminino e 17 do masculino, com o lado esquerdo sendo o predominante em 22 quadris (gráficos 1 e 2).

A média de idade dos pacientes no momento da cirurgia foi de 57,2 (27 a 77 anos). O seguimento médio foi de 8,3 anos (99,6 meses), sendo máximo de 12 anos e nove meses.

Os defeitos ósseos foram determinados de acordo com a classificação de D'Antonio, adotada pela American Academy of Orthopaedic Surgeons (AAOS) ${ }^{(3)}$ e a magnitude da cavidade acetabular, mensurada em milímetros (método dos $75 \mathrm{~mm}$ ), conforme idealizado pelo Serviço.

Em radiografias realizadas no pós-operatório imediato e tardio, comparou-se a migração do componente acetabular nos eixos horizontal e vertical. As linhas de radioluscência do acetábulo foram avaliadas de acordo com as áreas determinadas por DeLee et $a l^{(4)}$, em radiografias consecutivas, com o objetivo de visibilizar e mensurar possível progressão ${ }^{(5)}$. Nos pacientes cuja reconstrução foi realizada com bloco ósseo, avaliou-se a magnitude do mesmo, ou seja, se suportava mais de $50 \%$ ou menos de $50 \%$ da superfície do implante.

Os pacientes foram classificados clinicamente no pré e no pós-operatório pelo sistema de D'Aubignè et $a l^{(6)}$ modificado por Charnley et $a l^{(7)}$, de acordo com dor,
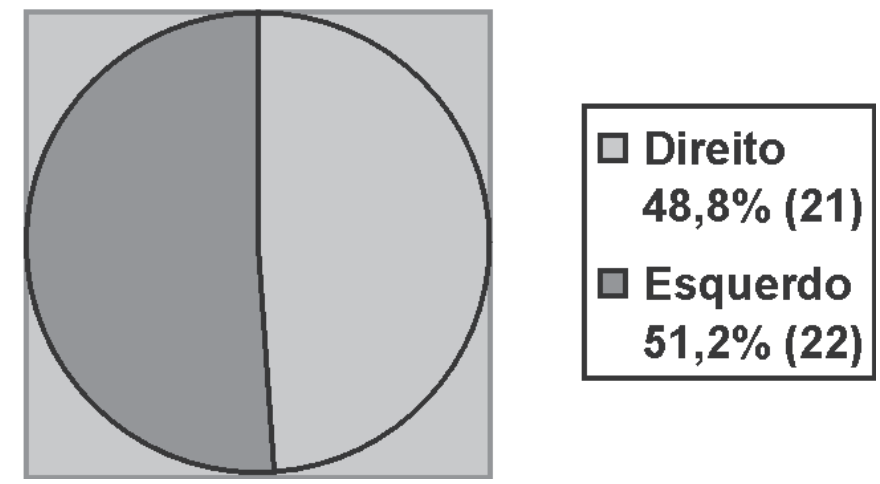

Gráfico 2 - Caracterização dos pacientes quanto ao lado acometido

qualidade da marcha e mobilidade. Consideraram-se resultados pós-operatórios ruins pacientes com escore menor do que 12; razoáveis, entre 13 e 14; bons, entre 15 e 16; e excelentes, entre 17 e 18. Resultados clínicos excelentes e bons foram classificados como satisfatórios; resultados razoáveis e ruins, como insatisfatórios.

Considerou-se falha da reconstrução devido a afrouxamento, a migração do implante maior do que $5 \mathrm{~mm}$ em qualquer direção, ou a progressão de linhas de radioluscência maior do que $2 \mathrm{~mm}$ de largura nas zonas delimitadas por DeLee et $a l^{(4)}$, em associação com o critério clínico de dor ${ }^{(1,8)}$.

A análise estatística foi realizada para se estabelecerem comparações entre as medidas do pré e do pósoperatório em relação aos escores que avaliam dor, marcha, mobilidade e escore geral, de D'Aubignè et $a l^{(6)}$ modificado por Charnley et $a l^{(7)}$, utilizando-se o teste de Wilcoxon. Compararam-se os grupos sucesso (sem critérios de falha da reconstrução devido a afrouxamento) e falha (com critérios de falha da reconstrução devido a afrouxamento) no que se refere à classificação AAOS do defeito acetabular e ao tipo de enxertia (enxerto em bloco ou picado), utilizando-se os testes "t" de Student e exato de Fisher. Os resultados foram considerados significativos quando apresentaram probabilidade de significância inferior a 5\% $(\mathrm{p}<0,05)$.

Realizou-se a curva de Kaplan-Meier, para análise de sobrevida cumulativa por mês das reconstruções, dos grupos de pacientes com enxerto em bloco e picado, somente em bloco e somente picado, com IC $95 \%$. 


\section{Avaliação radiográfica}

Todos os casos foram avaliados com radiografias ântero-posteriores de bacia com raio centrado na sínfise púbica e membros inferiores em rotação interna. Comparou-se a migração do componente acetabular no eixo horizontal e vertical em radiografias obtidas no pós-operatório imediato e tardio, utilizando como pontos de referência a gota de lágrima de ambos os quadris e um ponto médio do arco de metal do polietileno. Quando a gota de lágrima não era visível, utilizava-se a distância da linha obturadora e da linha de Köhler ao ponto médio do arco do implante ${ }^{(9)}$. O diâmetro da cabeça da prótese foi comparado com o diâmetro real em ambas as radiografias e, se houvesse discrepância, utilizava-se o fator de correção necessário.

Para evitar erros inter e intra-observadores, as aferições foram acompanhadas por dois cirurgiões do Grupo de Reconstrução do Quadril. No caso de haver discordância de mais de $3 \mathrm{~mm}$ na migração do componente acetabular, ou na progressão de linhas de radioluscência acetabulares, uma nova avaliação era executada, agora por um terceiro cirurgião, procedendo-se, então, a um consenso da aferição.

\section{Método dos 75mm}

A classificação idealizada para defeitos acetabulares é definida considerando-se o defeito maior ou menor do que $75 \mathrm{~mm}$. A aferição é feita em radiografia ântero-posterior de bacia medindo-se o rebordo ósseo externo da falha, superior e inferiormente. Se o defei- to for igual ou inferior a $75 \mathrm{~mm}$, indica-se reconstrução exclusivamente com osso picado "tipo crouton"; se for superior a $75 \mathrm{~mm}$, indica-se reconstrução com bloco ósseo associado a enxerto picado.

Este método de classificação foi definido preconizando-se que o acetábulo a ser utilizado nas reconstruções é cimentado, do tipo Charnley, tamanho 44, e com cabeça 22,25mm (espessura do polietileno de no mínimo $10 \mathrm{~mm}$ ); o manto de cimento deve ser de aproximadamente $3 \mathrm{~mm}$ e a espessura do enxerto picado não deve ultrapassar $15 \mathrm{~mm}$, para não comprometer a estabilidade da reconstrução.

Sabendo-se que a magnificação média de uma radiografia AP de bacia é de $15 \%{ }^{(10)}$, uma falha radiográfica acetabular de $75 \mathrm{~mm}$ corresponderá a um defeito real de $64 \mathrm{~mm}$. Portanto, subtraem-se de $64 \mathrm{~mm}$ o componente acetabular preconizado $(44 \mathrm{~mm})$ e o manto de cimento (aproximadamente $3 \mathrm{~mm}$ superior e $3 \mathrm{~mm}$ inferiormente), restando o que será preenchido, neste caso, com enxerto picado.

Os principais fatores que norteiam este método e a técnica cirúrgica subseqüente são leito receptor adequado à enxertia, resistência mecânica do enxerto e estabilidade mecânica inicial da reconstrução acetabular $^{(11-12)}$.

Assim se definiu uma padronização para as reconstruções acetabulares (figuras 1 e 2).

\section{Técnica cirúrgica}

Pré-operatoriamente, mensura-se o tamanho da falha acetabular e planeja-se o procedimento cirúrgico de acordo com método previamente citado.

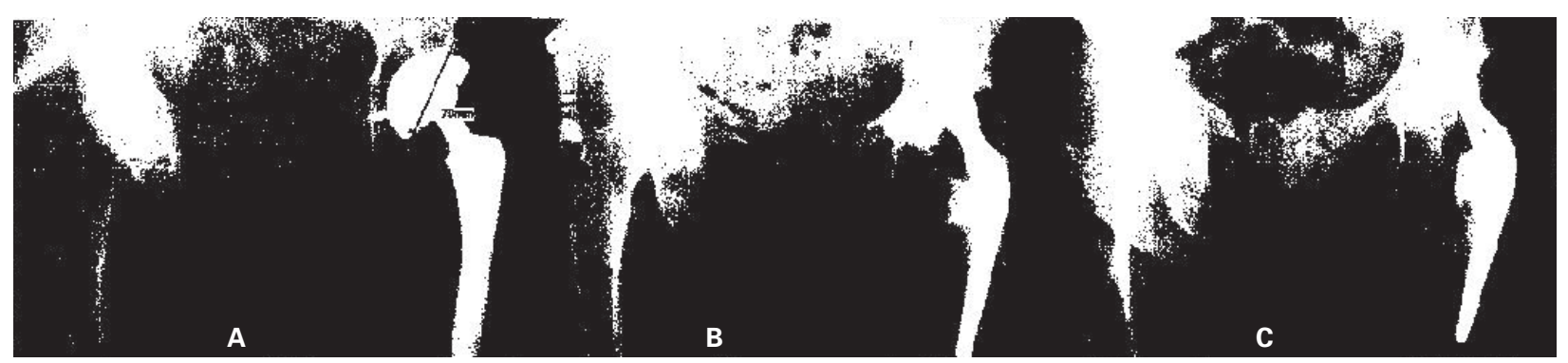

Figura 1 - a) Paciente de 74 anos, com defeito acetabular AAOS tipo II, de 70mm. b) Radiografia pós-operatória imediata, evidenciando reconstrução acetabular com enxerto ósseo picado. c) Aspecto radiográfico após 10 anos e 5 meses, com migração do implante de $1 \mathrm{~mm}$ lateral e $3 \mathrm{~mm}$ cranial. Paciente deambulando sem dor, escore de D'Aubignè e Postel modificado por Charnley de 18 pontos. 


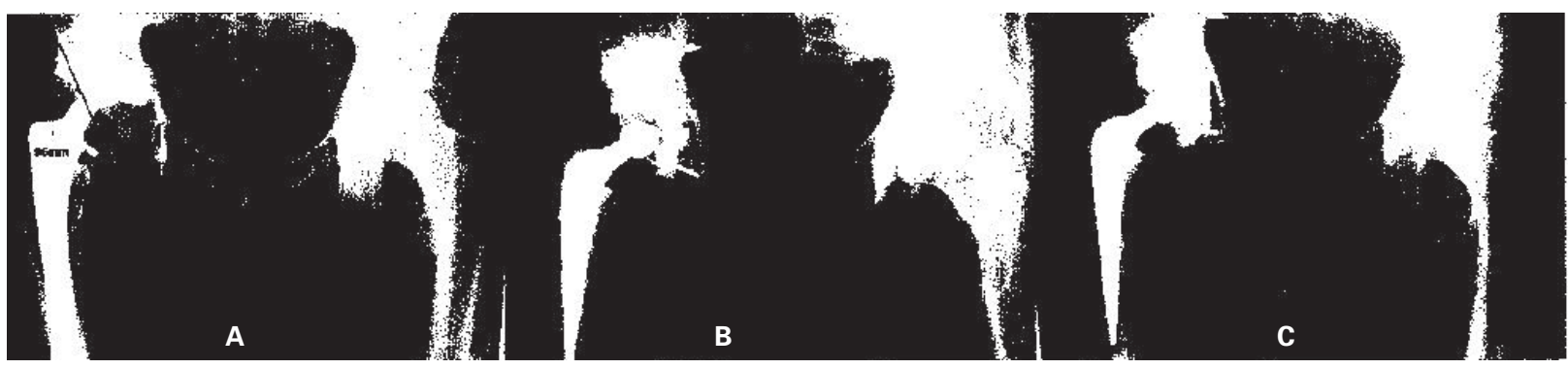

Figura 2 - a) Paciente de 58 anos, com defeito acetabular AAOS tipo III, 95mm. b) Radiografia pós-operatória imediata, evidenciando reconstrução acetabular com enxerto em bloco. c) Aspecto radiográfico após 6 anos e 3 meses, com migração do implante de $2 \mathrm{~mm}$ lateral. Paciente deambulando sem dor, escore de D'Aubignè e Postel modificado por Charnley de 18 pontos.

Utiliza-se a abordagem cirúrgica ântero-lateral de Hardinge modificada, em todos os casos. Inicialmente, realiza-se a remoção da haste, seguida do acetábulo; após, efetua-se a limpeza de debris e tecido fibroso da cavidade, utilizando curetas e fresas acetabulares, com o objetivo de alcançar uma superfície cruenta e apta a receber a enxertia.

Quando o defeito acetabular é menor ou igual a $75 \mathrm{~mm}$, utiliza-se exclusivamente enxerto picado "tipo crouton" ( $1 \mathrm{~cm}^{3}$ cada peça), para preenchimento do defeito. Com impactores manuais e fresas acetabulares no reverso, obtêm-se a estabilidade do enxerto (fundamental para o sucesso do procedimento) e o diâmetro ideal do acetábulo.

Quando o defeito acetabular é maior do que $75 \mathrm{~mm}$, utiliza-se enxerto em bloco no teto acetabular associado a enxerto picado. Após a limpeza do acetábulo, prepara-se um leito com enxerto picado impactado no local que receberá o bloco ósseo, para que não existam "zonas mortas" que permitam a formação de tecido fibroso ou cistos, dificultando a consolidação e possível integração do bloco. A fixação do enxerto estrutural no osso hospedeiro se dá por meio de parafusos esponjosos de $3,5 \mathrm{~mm}$ com rosca parcial, em ângulo de $45^{\circ}$ e em número variável de acordo com o tamanho e número de blocos ósseos utilizados. Não são empregados anéis de reforço mesmo em blocos de grandes dimensões. Após a fixação do enxerto, fresa-se a cavidade até que atinja o tamanho ideal.

Realizado o preenchimento complementar dos defeitos existentes, cimenta-se o acetábulo com ao menos uma ancoragem, feita superiormente com broca de $10 \mathrm{~mm}$, necessariamente alcançando o osso hospedeiro, e outra feita no ísquio através do osso hospedeiro, enxerto impactado, ou bloco ósseo (dependendo do tamanho da cavidade existente).

\section{Manejo pós-operatório}

Os pacientes são mantidos em repouso no leito por aproximadamente $48 \mathrm{~h}$ (com variações de acordo com as circunstâncias transoperatórias) e, após esse período, é permitida a posição sentada (fora do leito). Utiliza-se profilaxia tromboembólica mecânica no pós-operatório imediato, antiagregante plaquetário durante trinta dias e antibioticoterapia com vancomicina por 10 dias. É realizada avaliação radiográfica seis semanas após o procedimento cirúrgico. A partir desse momento, é permitido apoio total com uma muleta como elemento acessório de equilíbrio.

\section{RESULTADOS}

Do total de pacientes avaliados, $29(67,4 \%)$ foram submetidos à reconstrução acetabular com enxerto picado (defeito menor ou igual a $75 \mathrm{~mm}$ ) e os 14 casos $(32,6 \%)$ restantes, à reconstrução acetabular com enxerto em bloco (defeito maior de $75 \mathrm{~mm}$ ) (gráfico 3).

De acordo com a classificação da $\operatorname{AAOS}^{(3)}$, oito defeitos $(18,6 \%)$ foram considerados segmentares (tipo I); 16 (37,2\%), cavitários (tipo II); 14 (32,6\%), combinados (tipo III) e cinco (11,6\%), descontinuidade pélvica (tipo IV) (gráfico 4). Dos casos com defeito tipo I, sete reconstruções foram realizadas com enxerto picado $(87,5 \%)$ e uma, com enxerto em bloco $(12,5 \%)$; dos casos do tipo II, todas o foram com en- 

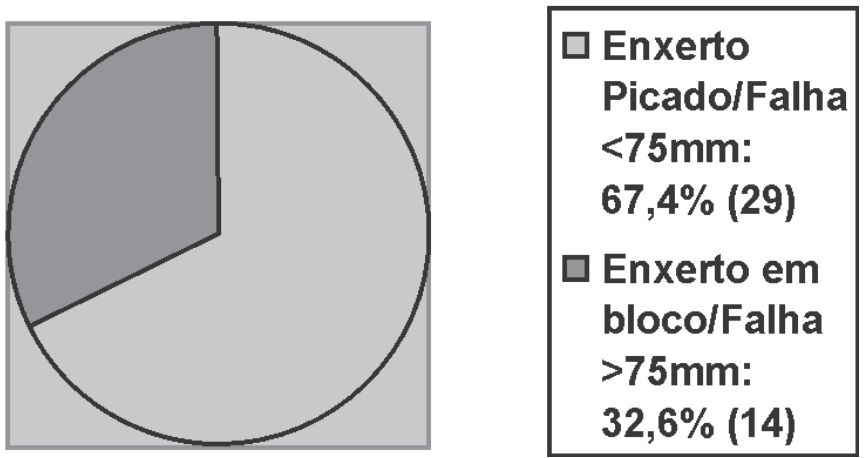

Gráfico 3 - Caracterização dos pacientes quanto ao tipo de enxertia utilizada e magnitude da falha $(\mathrm{mm})$

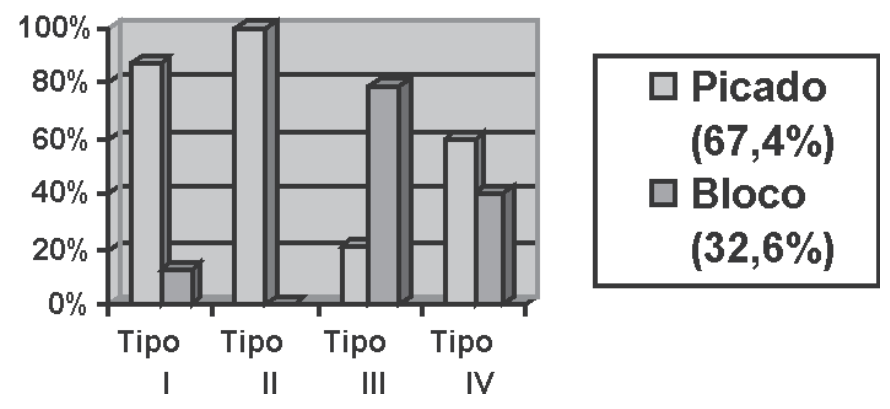

Gráfico 5 - Caracterização do tipo de defeito acetabular de acordo com AAOS e a enxertia utilizada

xerto picado (100\%); dos casos do tipo III, três foram feitas com enxerto picado $(21,4 \%)$ e 11 , em bloco $(78,6 \%)$; e dos do tipo IV, três o foram com enxerto picado (60\%) e duas, em bloco (40\%) (gráfico 5).

Considerando a mensuração da cavidade acetabular em milímetros (método dos $75 \mathrm{~mm}$ ), obteve-se média de 74,7mm (mínimo de 55mm e máximo de $95 \mathrm{~mm}$ ).

Com relação à avaliação da migração acetabular nos sentidos vertical e horizontal, oito pacientes $(18,6 \%)$ apresentaram migração maior que $5 \mathrm{~mm}$ em qualquer dos sentidos. A direção da migração do acetábulo mais encontrada foi cranial e lateral, em $62,7 \%$ dos pacientes em que houve migração.

Utilizando o critério de avaliação radiográfica das zonas de radioluscência maiores que $2 \mathrm{~mm}$ nas zonas de DeLee et $a l^{(4)}$, observaram-se oito casos $(18,6 \%)$ com linhas progressivas.

Considerando a magnitude dos blocos nos pacientes submetidos à reconstrução acetabular com este tipo de enxertia, observou-se que em somente um caso

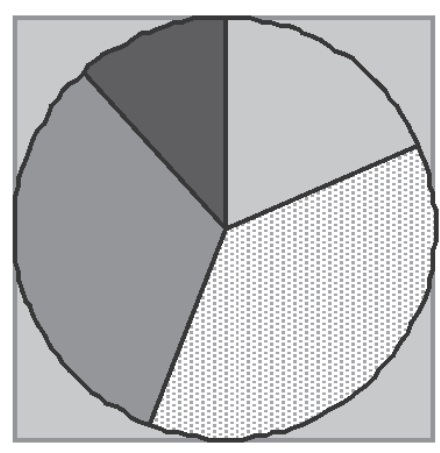

\begin{tabular}{|c|}
\hline$\square$ Segmentar \\
$18,6 \%(8)$ \\
Gavitário \\
$37,2 \%(16)$ \\
$\square$ Combinado \\
$32,6 \%(14)$ \\
$\square$ Desc. Pélvica \\
$11,6 \%(5)$ \\
\hline
\end{tabular}

Gráfico 4 - Caracterização dos pacientes quanto à classificação do defeito acetabular (AAOS)

(7,1\% de 14 pacientes) o bloco suportava menos de $50 \%$ da superfície do implante.

A classificação clínica pós-operatória, de acordo com os critérios de D'Aubignè et $a l^{(6)}$, modificado por Charnley et $a l^{(7)}$, considerou os resultados pós-operatórios obtidos como: 32 pacientes $(74,4 \%)$ com resultados excelentes; quatro pacientes $(9,3 \%)$, bons, e sete pacientes $(16,3 \%)$ ruins (todas falhas), com seguimento mínimo de seis anos. Portanto, obtiveram-se $83,7 \%$ de resultados clínicos satisfatórios. A avaliação dos resultados obtidos pelos critérios de D'Aubignè et $a l^{(6)}$, modificados por Charnley et $a l^{(7)}$, mostrou diferença estatisticamente significativa $(\mathrm{p}<0,05)$ entre as medidas pré e pós-operatórias no que tange aos escores de dor, marcha e mobilidade (gráficos 6, 7, 8 e 9).

Dessa maneira, sete pacientes $(16,3 \%)$ foram considerados como falhas de reconstrução devido a afrouxamento, todos apresentando migração maior do que $5 \mathrm{~mm}$, zonas de radioluscência progressivas maiores de $2 \mathrm{~mm}^{(5)}$ e quadro clínico de dor. As falhas ocorreram,
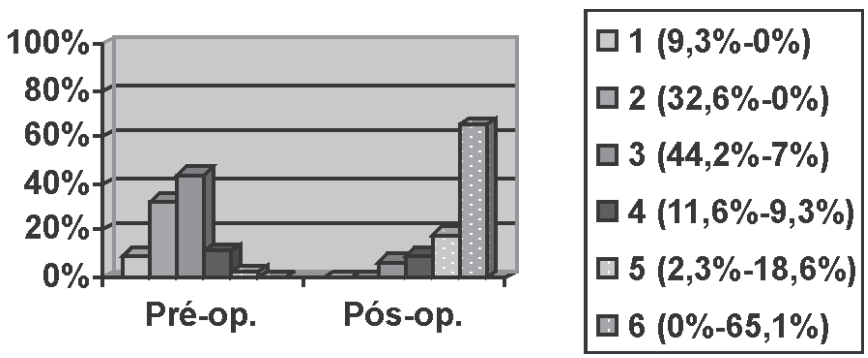

Gráfico 6 - Comparação entre as medidas do pré e do pós-operatório tardio em relação ao escore que avalia a dor 


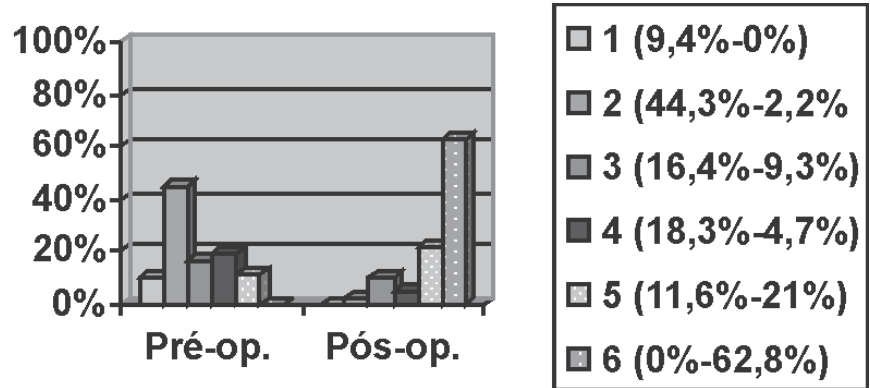

Gráfico 7 - Comparação entre as medidas do pré e do pós-operatório tardio em relação ao escore que avalia a marcha

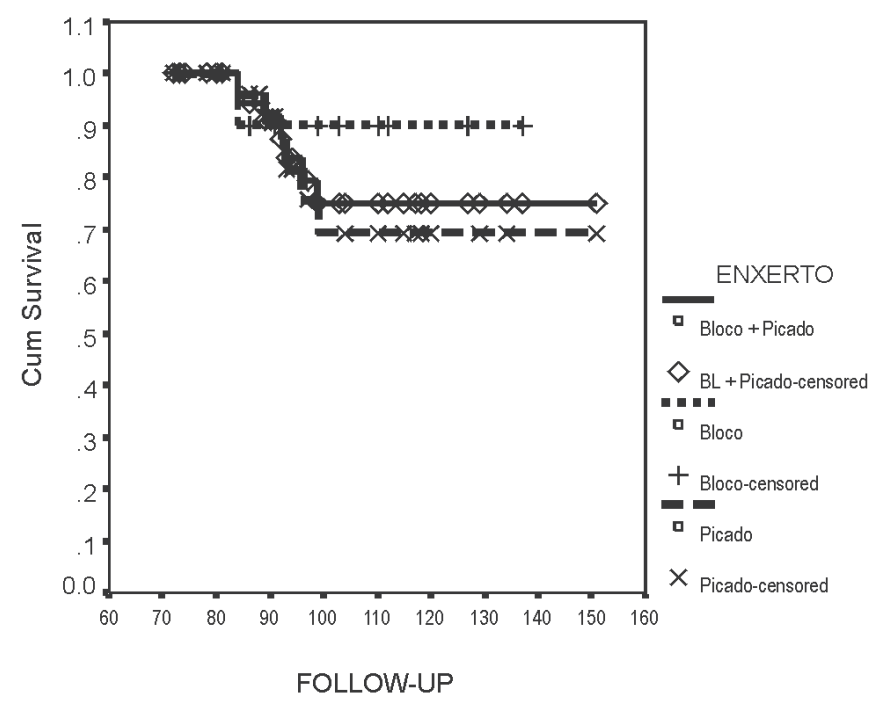

Gráfico 9 - Curva de Kaplan-Meier com IC 95\%, apresentando sobrevida cumulativa por mês da reconstrução dos grupos de pacientes com: enxerto em bloco e picado, somente em bloco, e somente picado.

em média, aos 7,25 anos (entre 72 e 110 meses), em seis casos de reconstrução com enxerto picado ( $20 \%$ das 29 enxertias com osso picado) e em um caso de reconstrução com enxerto em bloco $(7,1 \%$ dos 14 blocos). Considerando-se a classificação da AAOS, houve $25 \%$ de falha (dois casos) nos quadris com defeito tipo I, 12,5\% (dois casos) nos defeitos tipo II, 7,1\% (um caso) nos defeitos tipo III e $40 \%$ (dois casos) nos defeitos tipo IV. A análise estatística mostrou que não existe diferença estatisticamente significante $(\mathrm{p}>0,05)$ na comparação entre os grupos sucesso e falha no que se refere à classificação AAOS do defeito acetabular e ao tipo de enxertia (tabela 1). Não há como afirmar, neste estudo, se a ausência de diferença entre os gru-

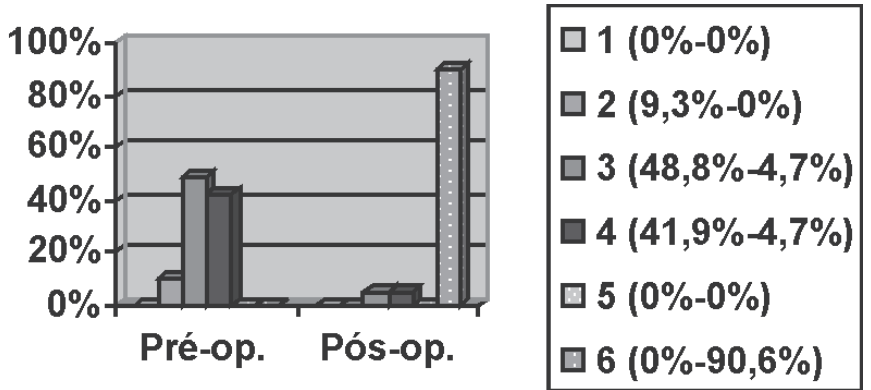

Gráfico 8 - Comparação entre as medidas do pré e do pós-operatório tardio em relação ao escore que avalia o arco de movimento

TABELA 1

Distribuição dos grupos de falha e sucesso em relação à Classificação AAOS do defeito acetabular $(p>0,05)$

\begin{tabular}{clrr}
\hline Classif. AAOS & Falha $(\mathbf{n}=\mathbf{7})$ & \multicolumn{2}{c}{ Sucesso $(\mathbf{n}=\mathbf{3 6})$} \\
\hline Tipo I & $2(28,6 \%)$ & 6 & $(16,7 \%)$ \\
Tipo II & $2(28,6 \%)$ & $14 \quad(38,9 \%)$ \\
Tipo III & $1(14,2 \%)$ & 13 & $(36,1 \%)$ \\
Tipo IV & $2(28,6 \%)$ & 3 & $(8,3 \%)$ \\
Total & $7(100 \%)$ & $36(100 \%)$ \\
\hline
\end{tabular}

pos decorreu em conseqüência de um número menor de casos de reconstrução com a utilização de bloco ósseo (tamanho da amostra) em comparação com enxerto picado, que, por conseguinte, poderia refletir em pouco poder estatístico.

De acordo com a metodologia aplicada, dois pacientes $(4,7 \%)$ apresentaram falhas radiográficas. Um deles, cujo acetábulo foi reconstruído com enxerto picado, apresentava zonas de radioluscência progressivas maiores que $2 \mathrm{~mm}$ na zona III de Delee et $a l^{(4)}$. Porém, este caso apresentava escore pós-operatório de D'Aubignè et $a l^{(6)}$, modificado por Charnley et $a l^{(7)}$, de 18 pontos (sem dor) e não havia migração acetabular. O outro paciente, cuja reconstrução acetabular foi realizada com bloco ósseo, apresentou migração de $6 \mathrm{~mm}$ no sentido cranial e $2 \mathrm{~mm}$ no sentido lateral; o escore foi de 17 pontos (também sem dor) e não havia zonas de radioluscência nas radiografias.

\section{DISCUSSÃO}

A reconstrução acetabular após soltura do implante associada à perda óssea sempre foi um desafio para o 
cirurgião de quadril. A literatura oferece inúmeras estratégias para a resolução desse problema, porém ainda não há consenso em relação à melhor técnica cirúrgica a ser realizada. Próteses de grandes dimensões, preenchimento com enxerto ósseo, elevação do centro de rotação, ressecção artroplástica e reconstrução com uso de enxertos ósseos são algumas das alternativas existentes $^{(2,8)}$.

As cirurgias de revisão das artroplastias de quadril com uso de enxerto ósseo podem ser realizadas por dois métodos, de acordo com o tamanho do fragmento a ser utilizado: forma picada ou bloco ósseo ${ }^{(2,8,13-14)}$. Jasty et al apresentaram bons resultados com o uso de enxertia em bloco. Paprosky et al reportaram taxa de falha de 19\% com seguimento médio de 5,7 anos com uso de blocos ósseos na reconstrução do quadril associado a acetábulo não cimentado(15). A utilização de grandes blocos ósseos com acetábulos cimentados, que suportam mais de $50 \%$ da superfície do implante, apresenta os piores resultados, com taxas de falhas de até $32 \%$ em seis anos de acompanhamento ${ }^{(14)}$. Pequenos blocos ósseos que suportam menos de $50 \%$ da superfície acetabular apresentam sobrevida de até 78,4\% em acompanhamento igual a $10 \operatorname{anos}^{(16)}$.

Em recente artigo de van Haaren et al, utilizando a técnica de enxerto picado e impactado associado a acetábulo cimentado e tela de reforço, a taxa de sobrevida em acompanhamento médio de 7,2 anos foi de $72 \%$, com falha ocorrendo principalmente em pacientes com defeitos tipo III e IV da AAOS ${ }^{(3,17)}$. Outros trabalhos identificaram inúmeras complicações precoces e grande migração do componente associado ao uso desta técnica ${ }^{(17-19)}$. Comba et al relataram sobrevida de $95,8 \%$ com o mesmo método, em acompanhamento médio curto de 51,7 meses (4,3 anos) em casos em que não havia defeito acetabular tipo IV da $\operatorname{AAOS}^{(20)}$. Sloof $e t$ al apresentaram taxa de $94 \%$ de bons resultados em acompanhamento médio de 12 anos, porém não foi especificada a espessura da camada ou a quantidade de enxertia utilizada nestas reconstruções ${ }^{(1)}$.

Os autores do presente estudo preconizam a reconstrução e restauração de forma biológica da biomecânica do quadril, o mais próximo do normal possível. Assim, o emprego do enxerto ósseo permite o uso de um acetábulo de tamanho normal, restaura o centro de rotação anatômico do quadril, corrige a discrepância de comprimento dos membros e repõe o estoque ósseo perdido. Por essas razões, este é o método de reconstrução acetabular de nossa escolha.

O principal determinante do tipo de enxerto a ser utilizado (bloco ou picado) é o tamanho do defeito acetabular, tendo como objetivo maior alcançar a melhor estabilidade inicial da reconstrução e resistência mecânica do enxerto (método dos $75 \mathrm{~mm}$ ). O uso de enxertia picada e impactada em defeitos de grandes dimensões provoca micromovimentos que levam à instabilidade mecânica e, conseqüentemente, à sua absorção ${ }^{(17-19)}$. Nesse caso, o uso de blocos ósseos propicia a estabilidade desejada.

A escolha do seguimento mínimo de seis anos como critério de exclusão ocorreu em virtude da observação clínica de que as falhas do método, quando constatadas, ocorriam em torno dos sete a oito anos do pósoperatório.

Foram utilizados critérios clínicos e radiográficos para a avaliação de sobrevida da reconstrução, e não o momento da nova revisão (como observado em diversos trabalhos) $)^{(17,20)}$, que por variados motivos (decisão do paciente sobre a realização da cirurgia, burocracia retardando a realização do procedimento, etc.), poderiam não expressar efetivamente esta análise.

Este trabalho, utilizando o método idealizado, relata sobrevida de $83,7 \%$ da reconstrução acetabular, com acompanhamento médio dos pacientes de 8,3 anos (IC $95 \%, 71,7 \%$ a $95,8 \%$ ). Analisando as curvas de Kaplan-Meier, observa-se que o grupo de pacientes com reconstrução acetabular utilizando bloco ósseo apresenta maior sobrevida que o grupo com enxerto picado. Também houve proporcionalmente maior número de falhas associadas a pacientes com defeito tipo descontinuidade pélvica (40\%), não havendo diferença estatisticamente significante entre os grupos compa$\operatorname{rados}(\mathrm{p}>0,05)$.

\section{CONCLUSÃO}

Com base nos resultados obtidos, conclui-se que o método apresentou alto índice de sobrevida da reconstrução acetabular e resultados clínicos satisfatórios em 
seguimento médio de 8,3 anos. Constatou-se maior sobrevida nos casos que foram realizados utilizando blocos ósseos, apesar de a cavidade ser de maior dimensão, observados os critérios de leito receptor adequado à enxertia, resistência mecânica do enxerto e estabilidade mecânica inicial da reconstrução, elementos todos já conhecidos da biologia do tecido ósseo.

\section{REFERENCIAS}

1. Sloof TJ, Buma P, Schreurs BW, Schimmel JW, Huiskes R, Gardeniers J. Acetabular and femoral reconstruction with impacted graft and cement. Clin Orthop Relat Res. 1996;(324): 108-15.

2. Devito FS, Aristides RSA, Honda EK, Chueire AG. O uso de enxerto homólogo na revisão de artroplastias do quadril com cimentação do componente acetabular. Acta Ortop Bras. 2006; 14(5):280-2.

3. D'Antonio JA. Periprosthetic bone loss of the acetabulum. Classification and management. Orthop Clin North Am. 1992; 23(2):279-90.

4. DeLee JG, Charnley J. Radiological demarcation of cemented sockets in total hip replacement. Clin Orthop Relat Res. 1976; (121):20-32.

5. Reckling FW, Asher MA, Dillon WL. A longitudinal study of the radiolucent line at the bone-cement interface following total joint-replacement procedures. J Bone J Surg Am. 1977; 59(3): $355-8$.

6. D'Aubigne RM, Postel M. Function al results of hip arthroplasty with acrylic prosthesis. J Bone Joint Surg Am. 1954;36-A(3):451-75.

7. Charnley J, Cupic Z. The nine and ten year results of the lowfriction arthroplasty of the hip. Clin Orthop Relat Res. 1973; (95):9-25.

8. Schreurs BW, Slooff TJ, Gardeniers JW, Buma P. Acetabular reconstruction with bone impaction grafting and a cemented cup: 20 years' experience. Clin Orthop Relat Res. 2001;(393): 202-15.

9. Massin P, Schmidt L, Engh CA. Evaluation of cementless acetabular component migration. An experimental study. J Arthroplasty. 1989;4(3):245-51.
10. Clarke IC, Gruen T, Matos M, Amstutz HC. Improved methods for quantitative radiographic evaluation with particular reference to total-hip arthroplasty. Clin Orthop Relat Res. 1976;(121):83-91.

11. Chagas AM, Camisa Júnior A, Dozza PR, Roos MV. O enxerto ósseo homólogo de banco em cirurgia de revisão com prótese total de quadril não-cimentada. Rev Bras Ortop. 1993;28(5): 309-14.

12. Roos MV, Camisa Júnior A. A reconstrução acetabular com enxerto homólogo na revisão com prótese cimentada: resultados tardios. Rev Bras Ortop. 1994;29(6):398-400.

13. Gross AE, Allan DG, Catre M, Garbuz DS, Stockley I. Bone grafts in hip replacement surgery. The pelvic side. Orthop Clin North Am. 1993;24(4):679-95.

14. Jasty M, Harris WH. Salvage total hip reconstruction in patients with major acetabular bone deficiency using structural femoral head allografts. J Bone Joint Surg Br. 1990;72(1):63-7.

15. Paprosky WG, Perona PG, Lawrence JM. Acetabular defect classification and surgical reconstruction in revision arthroplasty. A 6-year follow-up evaluation. J Arthroplasty. 1994;9(1):33-44.

16. Woodgate IG, Saleh KJ, Jaroszynsky G, Agnidis Z, Woodgate MM, Gross AE. Minor column structural acetabular allografts in revision hip arthroplasty. Clin Orthop Relat Res. 2000; (371):75-85.

17. van Haaren EH, Heyligers IC, Alexander FG, Wuisman PI. High rate of failure of impaction grafting in large acetabular defects. J Bone Joint Surg Br. 2007;89(3):296-300.

18. Ornstein E, Atrashi I, Franzén H, Johnsson R, Sandquist P, Sundberg M. Early complications after one hundred and fortyfour consecutive hip revisions with impacted morselized allograft bone and cement. J Bone J Surg Am. 2002;84-A(8): 1323-8.

19. Nelissen RG, Valstar ER, Pöll RG, Garling EH, Brand R. Factors associated with excessive migration in bone impaction hip revision surgery: a radiostereometric analysis study. J Arthroplasty. 2002;17(7):626-33.

20. Comba F, Buttaro M, Pusso R, Piccaluga F. Acetabular reconstruction with impacted bone allografts and cemented acetabular components: a 2- to 13-year follow-up study of 142 aseptic revisions. J Bone Joint Surg Br. 2006;88(7):865-9. 